\title{
Caracterización y clasificación geomecánica del depósito de cobre localizado en el corregimiento de Camperucho, municipio de Valledupar, Cesar
}

Luis Araujo', Yasuana Britto², Keyla Meza³, J. Olivella ${ }^{4}$

\section{Resumen}

En las obras mineras, principalmente en aquellas a cielo abierto, se realizan numerosas excavaciones que requieren el diseño y control de los taludes abiertos; para este control se requiere explicar el comportamiento geomecánico del terreno, al igual que conocer las características de resistencia de este, para luego poder implementar los equipos necesarios para la seguridad y el rendimiento de la extracción. Este artículo presenta el estudio de las propiedades físicas y mecánicas del depósito de cobre perteneciente a la formación Guatapurí (Tgr), localizado en la vereda Camperucho, corregimiento de Mariangola, municipio de Valledupar, Cesar. Para la realización de la clasificación geomecánica se tomaron datos de 548 diaclasas en los tres frentes de explotación en el que se encuentra divida la zona de estudio. En el laboratorio se determinaron las características físico-mecánicas de las rocas. Al tabular la información y obtener los resultados del software dips se determinaron tres familias de discontinuidades, de las cuales se observan los diagramas de polos y la concentración de los mismos; además, luego de obtener el criterio de rotura de Hoek-Brown, se realizó la caracterización global, la cual determino que este macizo presenta una calidad media.

Palabras clave: estabilidad de taludes, mecánica de rocas, minería cielo abierto.

\footnotetext{
1 Ingeniero de minas de la Fundación Universitaria del Área Andina, programa Ingeniería de Minas. Valledupar, Colombia. Correo: laraujodareandina.edu.co

2 Ingeniera de minas de la Fundación Universitaria del Área Andina, programa Ingeniería de Minas. Valledupar, Colombia. Correo: yabrittodareandina.edu.co

3 Ingeniera de minas de la Fundación Universitaria del Área Andina, programa Ingeniería de Minas. Valledupar, Colombia. Correo: kemeza@areandina.edu.co

4 Ingeniero de minas de la Fundación Universitaria del Área Andina, programa Ingeniería de Minas. Valledupar, Colombia. Correo: joolivelladareandina.edu.co
} 


\section{Introducción}

El área de estudio se encuentra en el borde oriental de la Sierra Nevada de Santa Marta, básicamente está constituida por materiales aluviales del río Garupal que cubren las rocas antiguas vulcanosedimentarias de edad jurásica, correlacionables con la formación Guatapurí y rocas plutónicas (Batolito de Pueblo Bello). En la zona del corregimiento de Caracolí afloran, en su mayor parte, intrusivos pertenecientes a las rocas volcánicas ignimbríticas (Jvi) y riolitas (JKvr). Las rocas sedimentarias que afloran hacen parte de la formación Guatapurí (Tgr) y la formación Cogollo. La nomenclatura geológica utilizada corresponde a la propuesta por Tschanz y otros (1965).

- Rocas sedimentarias. Formación Guatapurí (Tgr). Esta formación aflora en la parte media del río Guatapurí, de donde toma su nombre, y desde este sitio hasta el arroyo las Lajas cerca de la carretera Valledupar, Bosconia. La unidad puede dividirse en dos conjuntos: una parte predominante volcánica, con algunas intercalaciones sedimentarias y una parte superior compuesta por limolitas rojizas a violáceas con intercalaciones tobáceas y de otras rocas pertenecientes a la parte superior de la formación.

- Formación Cogollo (Kmc). Según un estudio realizado por Ingeominas (1994) "esta formación se encuentra en la región sur de la Sierra Nevada constituido por calizas en un $60 \%, 15 \%$ de caliza arcillosa y $25 \%$ de lutita".

\section{Geología del yacimiento}

Las manifestaciones minerales de cobre se encuentra presentes en el área de estudio están representadas por malaquita con asociaciones de azurita, rellenando fracturas de rocas extrusivas de tipo riolítico y tobáceo y brechas poco o nada alteradas con poca mineralización de sulfuros con cobre nativo diseminado y cuarzo lechoso, las fracturas mineralizadas tienen una dirección preferencial al NW con inclinaciones de hasta 90 grados y están asociadas principalmente al intenso fracturamiento del área, influenciados por la falla Bucaramanga-Santa Marta y la falla en dirección NE como son la falla Cesarito al sur y la falla Maizmorocho; al norte se encuentran afectando rocas sedimentarias pertenecientes a la formación Guatapurí y Cogollo (Trabajos de Exploración y Programa de Trabajos y Obras, 2012). 


\section{Materiales y métodos}

\section{Localización}

Localmente el proyecto se encuentra ubicado en las partes adyacentes al carreteable que comunica al municipio de Valledupar con el municipio de Bosconia, a la altura del sector de Caracolí de la vereda Camperucho, localidad situada en el valle oriental del río Cesar, a unos $50 \mathrm{~km}$ de Valledupar.

Figura 1. Localización del área de estudio.

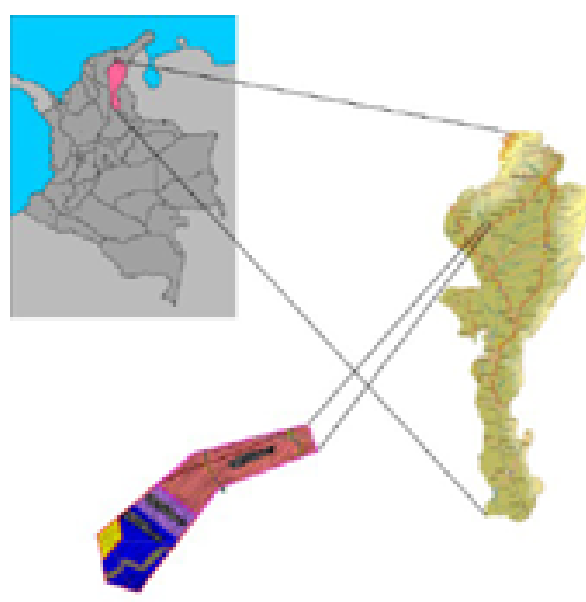

\section{Procedimiento}

La caracterización geomecánica se realizó basándose en los datos obtenidos en los trabajos de campo y con los resultados de los ensayos de laboratorios. El depósito de cobre está divido en tres frentes de explotación, en cada frente de se hizo un muestreo aleatorio simple de las muestras para ser llevadas al laboratorio. En estos tres frentes se realizó una descripción de las características generales del afloramiento, la cual consideró la identificación de las características generales del afloramiento y sus respectivos componentes. En cada zona se realizó una descripción general.

Para los ensayos de laboratorio se recolectaron las muestras directamente del macizo. Con estas muestras se llevó a cabo la ejecución del ensayo de compresión simple, para el cual se llevaron 6 muestras al laboratorio, teniendo en cuenta las normas: ASTM d3148-02 y ASTM d454; además, se realizó la prueba de desgaste utilizando la máquina de los ángeles, con el fin de determinar resistencia a la trituración o abrasión de la roca presente en la zona de estudio. Todo esto regido por la norma INV e-218.

Luego de los ensayos de laboratorio se realizó una descripción detallada de cada zona, enumerando las propiedades físicas y mecánicas del macizo rocoso y de las discontinuidades, midiendo aspectos como el número de familias de discontinuidades, orientación y características representativas de las mismas; para esto se utilizó el software dips. 
Tabla 1. Valores obtenidos en la resistencia a la compresión simple

\begin{tabular}{|l|l|l|}
\hline $\begin{array}{l}\text { Muestra } \\
\text { n. }\end{array}$ & $\begin{array}{l}\text { Resistencia la compresión } \\
\text { simple (MPA) }\end{array}$ & $\begin{array}{l}\text { Tipo de } \\
\text { rotura }\end{array}$ \\
\hline 1 & 3 & A \\
\hline 2 & 7 & B \\
\hline 3 & 7 & A \\
\hline 4 & 16 & E \\
\hline 5 & 44 & B \\
\hline 6 & - & - \\
\hline
\end{tabular}

Figura 2. Clases de rotura de la roca

En la caracterización global del macizo rocoso se proporcionan las condiciones geológicas y geomecánicas del macizo en su conjunto. Esto para determinar los parámetros de resistencia de masas de la roca, datos que son ingresados en el programa RocLab, el cual está basado en la última versión del criterio de falla generalizado de Hoek-Brown. Algunos de los datos necesarios para parametrizar el software RocLab fueron el tipo de roca presente en el área de estudio, en este caso se escoge la rolita ya esta es la roca caja de los minerales asociados al cobre como lo son la azurita y la malaquita. Por último, y teniendo en cuenta el dimensionamiento geométrico del talud, se determina el factor de seguridad con la ayuda del software Slide de Rocscience.

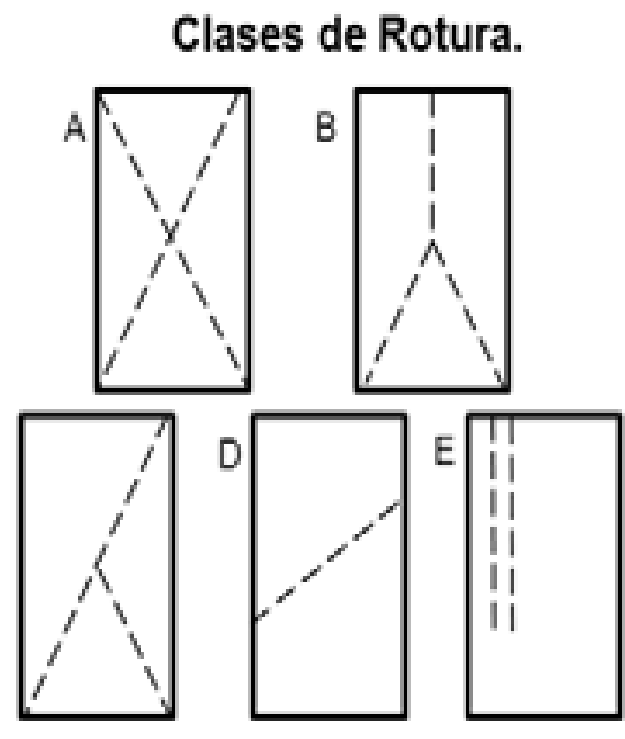

\section{Resultados}

Las características identificadas en este estudio son las que definen el esfuerzo máximo que soporta la roca, la resistencia a la abrasión y la clase a la 
que pertenece según la clasificación RMR.

\section{Esfuerzo máximo}

Para el cálculo del esfuerzo, se realizó el ensayo a compresión simple a seis muestras, el cual arrojó como resultado:

$$
x=\frac{16+3+7+7+44}{5}=15.4 \mathrm{MPA}
$$

\section{Resistencia al desgaste}

De esta prueba se obtuvo que el porcentaje de desgaste de la muestra fue del $22,43 \%$. Este porcentaje, según las normas Invías E-218 y E-219, es una roca abrasiva, que se puede utilizar como material de base granular con un porcentaje de arena equivalente al $30 \%$.

Luego de obtener las propiedades físicas de la roca, pasamos a realizar la clasificación RMR, que fue desarrollada por Bieniawski (1973), "la cual constituye un sistema de clasificación de macizos rocosos que permite relacionar índices de calidad con parámetros geotécnicos del macizo". Los parámetros son los relacionados a continuación:

- Resistencia a la compresión uniaxial: 15,4 MPa

- Índice de calidad de la roca (RQD): $75-90 \%$
- Separación entre diaclasas: $<0,006 \mathrm{~m}$

- Estado de las discontinuidades:

- Longitud de la discontinuidad: $<1 \mathrm{~m}$

- Abertura: $<0,01 \mathrm{~mm}$

- Rugosidad: ligeramente rugosa

- Relleno: ninguno

- Alteración ligeramente alterada.

- Presencia de agua: seco.

\section{Comportamiento de las familias de discontinuidades}

Para realizar este análisis se tuvo en cuenta las distintas diaclasas tomadas en el área de estudio (548), con las cuales, a través del software dips de Rocscience, se analizaron las principales familias de discontinuidades presentes en esta área y se obtuvieron las siguientes gráficas:Con el valor obtenido del RMR, las direcciones de las discontinuidades y del talud se procede a realizar la clasificación SMR, la cual es una corrección del RMR, propuesta por Romana (1985) en 1985, para predecir el comportamiento de los taludes en roca. La expresión del SMR es la siguiente: 
Figura 3. Polos

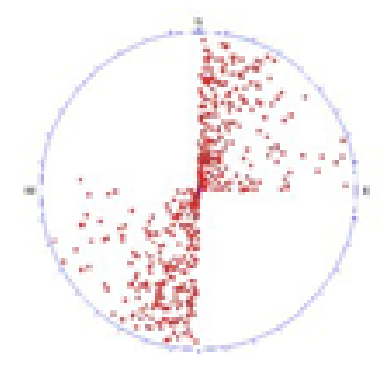

Figura 4. Discontinuidades

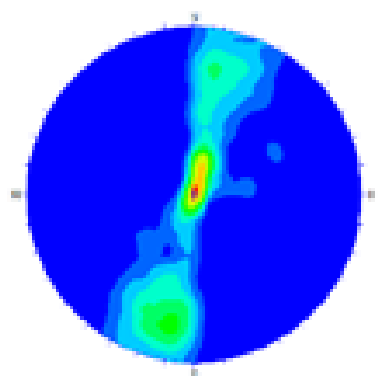

Figura 5. Talud

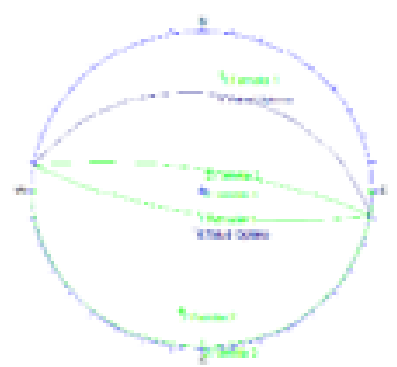

\section{Resultados}

- $\mathrm{F} 1=[1-\operatorname{SEN}(73-27)]$

- $\quad \mathrm{F} 1=0,28$

- $\quad \mathrm{F} 2=\mathrm{TAN}(73)^{2}$

- $\quad \mathrm{F} 2=4.01$

- $\quad \mathrm{F} 3=\mathrm{P}=73-27=46$

$\mathrm{T}=73+27=100$

$46 / 100=0,46$

- $\quad \mathrm{F} 4=0$

F4 es cero debido a que en la excavación mecánica de los taludes por ripado solo es posible cuando el macizo rocoso está muy fracturado o la roca blanda.

Con frecuencia se combina con prevoladuras poco cuidadas. Las caras del talud presentan dificultades de acabado. Por ello, el método ni mejora ni empeora la estabilidad.

\section{Rotura de la roca}

Como resultado de la parametrización del software RocLab con las condiciones geológicas y geomecánicas del macizo se obtuvo las siguientes características geomecánicas del macizo 
Tabla 2. Criterio de rotura de Hoek Brown obtenidos en el software RocLab

\begin{tabular}{|l|l|}
\hline \multicolumn{1}{|c|}{ Descripción } & \multicolumn{1}{c|}{ Valor } \\
\hline Resistencia a compresión simple & $15,4 \mathrm{MPa}$ \\
\hline Constante de Hoek y Brown & 25 \\
\hline Índice de esfuerzo geológico & 65 \\
\hline Ángulo de fricción interna & $58,44^{\circ}$ \\
\hline Cohesión & $0,188 \mathrm{Mpa}$ \\
\hline Esfuerzo de tensión & $-0,026$ \\
\hline Módulo de deformación & $6048,86 \mathrm{Mpa}$ \\
\hline
\end{tabular}

En las muestras 4 y 5 del ensayo de compresión simple se observan diferencias significativas respecto al resultado obtenido, en comparación a los obtenidos del resto de las muestras; las diferencias se deben a que cuando la roca presenta anisotropía su resistencia compresiva para un mismo esfuerzo varía según el ángulo $\beta$ entre la dirección de los planos de anisotropía y la dirección de la carga aplicada, pudiendo presentar valores muy diferentes (figura 6).
Figura 6. Anisotropía en rocas

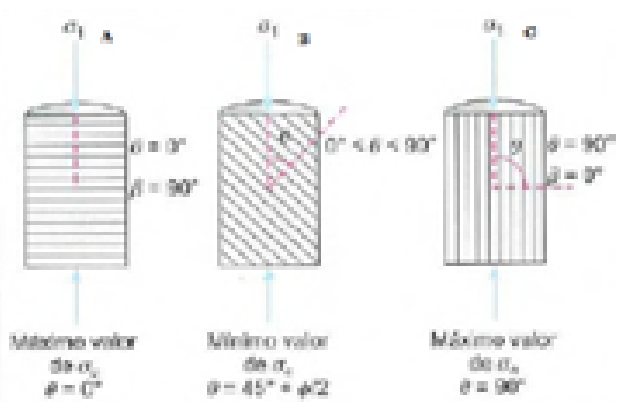

Fuente: González de Vallejo, L., Ferrer, M., Ortuño, L. y Oteo, C. (2002, p. 159).

En las muestras que presenten una dirección de planos como la figura $6 \mathrm{~A}$ presentan menor resistencia en comparación con aquellas que presentaron una dirección de planos de la figura 6C.

En cuanto a la muestra número 6, podemos indicar que los planos de debilidad preexistentes en esta muestra no permitieron realizar el ensayo dado que 
estos controlan los procesos de deformación y dividieron la muestra al momento de hacer la extracción del núcleo

En el análisis de las familias de discontinuidades se obtuvieron tres familias de, que a partir del análisis cinemático mediante proyección estereográfica se deduce que la inestabilidad se presenta por una rotura de cuña.

Figura 6. Rotura en cuña
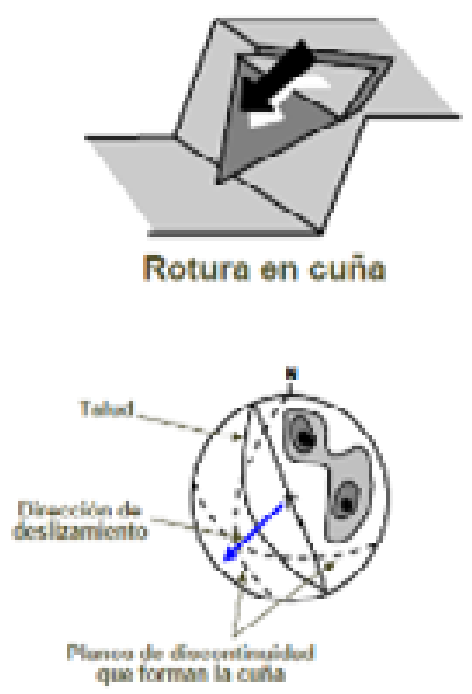

Teniendo en cuenta estos resultados y según la Sociedad Internacional de Mecánica de Rocas, encontramos una roca blanda, dado que su resistencia a la compresión simple es de 15,4 Mpa, y, según la clasificación RMR, el terreno se clasifica como un macizo rocoso de clase III, el cual se considera un macizo de calidad media, con un valor final en la evaluación de 59 .

\section{Conclusiones}

Dadas las condiciones del terreno, las condiciones más favorables para el talud serían una altura de banco máxima de diez metros, con un ancho de la vía de siete metros y un ángulo de fricción interna de $58,44^{\circ}$.

Teniendo en cuenta el dimensionamiento geométrico de talud, y luego de realizar la modelación del talud en el programa Slide de Rocscience, se obtuvo como resultado un factor de seguridad de 2,079, usando el método de Bishop simplificado.

Dada la clasificación SMR una estabilidad de clase III. Este talud es normal, parcialmente estable y con desarrollo de muchas cuñas. El valor de SMR obtenido indica, además, que el talud necesita un tratamiento de carácter sistemático (Geología de Mayorca, 2006).

Debido a las condiciones del terreno y los resultados del ensayo de la compresión simple, además, teniendo en cuenta los parámetros establecidos por el Instituto Geológico Minero de España (IGME) en el libro Manual de perforación y voladuras en roca (López Jimeno, 
Ayala-Carcedo, y Pernía Llera, 1994), se debe realizar una voladura de pequeño diámetro con las siguientes variables:

- Diámetro de barreno: $0.065 \mathrm{~m}$

- Burden: 2,53 m

- Espaciamiento: 3,31 m

- Retacado: 2,27 m

- Sobreperforación: 0,65 m

Debido a la topografía de la zona de estudio y a que los yacimientos de cobre muchas veces no tienen rumbo ni buzamiento definido, el método de explotación que se puede aplicar es el método de cortas, con un botadero en el exterior.

El método de cortas, a pesar de tener una afectación ambiental tipo media-alta, por los estériles que se deben manejar, la restauración ambiental se puede iniciar en un periodo a mediano plazo, lo que lo convierte en un método seguro y amigable con el medio ambiente (Araujo, 2013).

\section{Referencias}

Araujo, L. (2013). Programa de trabajos y obras contrato de concesión HEF-152.

Bieniawski, Z. T. (1973). The geomechanics lassification of rock engineering application.
Carrillo, M., Linkimer, L., Rodríguez, A. y Zúñiga, M. (1999). Clasificación geomecánica y análisis de estabilidad de taludes de macizos rocosos. Costa Rica: Universidad de Costa Rica. Recuperado de http://www. geologia.ucr.ac.cr/revista/to_pdf/revista/26/26-CARRILLO.pdf

Cartaya, M. (2006). Caracterización geomecánica de macizos rocosos en obras subterráneas de la región oriental del país. (Tesis doctoral). Recuperada de la base de datos Redalyc.

Ferre, M. y González de Vallejo, L. (2007). Manual de campo para la descripción y caracterización de macizos rocosos en afloramientos. Madrid: Instituto Geológico y Minero de España.

Geología de Mayorca (2006). Estudio de estabilidad de Taludes. Cantera Coma de S'aigua,

González de Vallejo, L., Ferrer, M., Ortuño, L. y Oteo, C. (2002). Ingeniería geológica. Madrid: Pearson Educación.

Ingeominas (1994). Evaluación del agua subterránea en el departamento del Cesar. Informe Geológico. Bogotá, D. C.

Instituto Colombiano de Geología y Minería. (2010). Exploración de materiales lapidarios en la Sierra Nevada de Santa Marta, departamentos del Cesar y La Guajira.

López Jimeno, E., Ayala-Carcedo, F. y Pernía Llera, J. (1994). Manual de perforación y voladura de rocas. España: Instituto Geológico y Minero de España. 
Páez, H. (2010). La mecánica de rocas en la ingeniería de minas. Apuntes de mecánica de rocas. México: Universidad Autónoma de Chihuahua.

Romana, M. (1985). New adjustment ratings for application of Bieniawski classification to slopes. Int. Symp.

Trabajos de Exploración y Programa de Trabajos y Obras. Contratos de Concesión LJJ09471 y LJJ- 09211. 